2000]Primary 46B04; secondary 46B08, 46B20, 46M07

\title{
NARROW OPERATORS AND THE DAUGAVET PROPERTY FOR ULTRAPRODUCTS
}

\author{
DMITRIY BILIK, VLADIMIR KADETS, ROMAN SHVIDKOY, \\ AND DIRK WERNER
}

\begin{abstract}
We show that if $T$ is a narrow operator (for the definition see below) on $X=X_{1} \oplus_{1} X_{2}$ or $X=X_{1} \oplus_{\infty} X_{2}$, then the restrictions to $X_{1}$ and $X_{2}$ are narrow and conversely. We also characterise by a version of the Daugavet property for positive operators on Banach lattices which unconditional sums of Banach spaces inherit the Daugavet property, and we study the Daugavet property for ultraproducts.
\end{abstract}

\section{INTRODUCTION}

A Banach space $X$ is said to have the Daugavet property if every rank-1 operator $T: X \rightarrow X$ satisfies

$$
\|\mathrm{Id}+T\|=1+\|T\| .
$$

Examples include $C[0,1], L_{1}[0,1]$, certain function algebras such as the disk algebra or $H^{\infty}$ and also their noncommutative counterparts (nonatomic $C^{*}$ algebras and preduals of nonatomic von Neumann algebras [7]). Such spaces are studied in detail in [5].

It has long been known that the $\ell_{1}$-sum and the $\ell_{\infty}$-sum of two spaces with the Daugavet property again enjoys the Daugavet property; see [1], [8] and [5] for various degrees of generality in this statement. In this paper we present an operator theoretic extension of this result along with a study of the inverse problem of which unconditional direct sums inherit the Daugavet property. Our methods are naturally related to ultrapower techniques, so we also study the Daugavet property for ultraproducts of Banach spaces.

In [6] we have introduced the notions of a strong Daugavet operator and of a narrow operator between Banach spaces $X$ and $Y$; for definitions see Section 2. These appear to be, in the case $X=Y$, the largest reasonable classes of operators that satisfy (1.1); in particular, weakly compact operators, operators not fixing a copy of $\ell_{1}$, strong Radon-Nikodým operators and their sums are strong Daugavet operators on Banach spaces with the Daugavet property. In Section 3 we show that an operator $T$ on $X_{1} \oplus_{\infty} X_{2}$ is a strong Daugavet operator if and only if both restrictions of $T$ to $X_{1}$

1991 Mathematics Subject Classification. [.

Key words and phrases. Daugavet property, narrow operator, strong Daugavet operator, ultraproducts of Banach spaces.

The work of the second-named author was supported by a grant from the Alexandervon-Humboldt Stiftung. 
resp. $X_{2}$ are strong Daugavet operators; the same is true for narrow operators. Section 4 studies the same problem on $\ell_{1}$-sums $X_{1} \oplus_{1} X_{2}$; here a subtle difference between strong Daugavet operators and narrow operators is exhibited, since the restriction of a narrow operator turns out to be narrow again, but the analogue for strong Daugavet operators proves to be false.

Section 5 deals with the inverse problem to determine which 1-unconditional sums of Banach spaces inherit the Daugavet property. These are completely classified, and it turns out that among all 1-unconditional sums of two spaces only $X_{1} \oplus_{1} X_{2}$ and $X_{1} \oplus_{\infty} X_{2}$ are spaces with the Daugavet property.

As pointed out above, our methods rely on ultrapower techniques as explained in Section 2. Thus it appears natural to try and investigate which ultrapowers $X^{\mathcal{U}}$ have the Daugavet property. These can be characterised by means of a quantitative version of the Daugavet property for $X$ that we call the uniform Daugavet property. As examples we show that $C[0,1]$ and $L_{1}[0,1]$ have the uniform Daugavet property.

We consider real Banach spaces in this paper; $S(X)$ stands for the unit sphere of a Banach space $X$ and $B(X)$ for its closed unit ball.

\section{The Rigid Versions of the Daugavet property, Strong DAUGAVET AND NARROW OPERATORS} [5]:

We recall the following characterisation of the Daugavet property from

Lemma 2.1. The following assertions are equivalent:

(i) $X$ has the Daugavet property.

(ii) For every $x \in S(X), x^{*} \in S\left(X^{*}\right)$ and $\varepsilon>0$ there exists some $y \in S(X)$ such that $x^{*}(y) \geq 1-\varepsilon$ and $\|x+y\| \geq 2-\varepsilon$.

An a bit more general notion will be convenient for us.

Definition 2.2. A Banach space $X$ has the Daugavet property with respect to a subset $\Gamma \subset S\left(X^{*}\right)(X \in \mathrm{DP}(\Gamma)$ for short) if for every $x \in S(X)$, $x^{*} \in \Gamma$ and $\varepsilon>0$ there exists some $y \in S(X)$ such that $x^{*}(y) \geq 1-\varepsilon$ and $\|x+y\| \geq 2-\varepsilon$.

According to [6] an operator $T$ on a Banach space $X$ is said to be a strong Daugavet operator if for every two elements $x, y \in S(X)$ and for every $\varepsilon>0$ there is an element $z \in S(X)$ such that $\|x+z\| \geq 2-\varepsilon$ and $\|T(y-z)\| \leq \varepsilon$. We denote the set of all strong Daugavet operators on $X$ by $\mathcal{S D}(X)$. An operator $T$ is said to be a narrow operator if for every two elements $x, y \in S(X)$, for every $x^{*} \in X^{*}$ and for every $\varepsilon>0$ there is an element $z \in S(X)$ such that $\|x+z\| \geq 2-\varepsilon$ and $\|T(y-z)\|+\left|x^{*}(y-z)\right| \leq \varepsilon$. We denote the set of all narrow operators on $X$ by $\mathcal{N A R}(X)$.

To indicate the difference between the two classes we have introduced the following notation in [6]. If $T: X \rightarrow Y$ is an operator and $x^{*}: X \rightarrow \mathbb{R}$ is a 
functional, define

$$
T \tilde{+} x^{*}: X \rightarrow Y \oplus_{1} \mathbb{R}, \quad x \mapsto\left(T x, x^{*}(x)\right) .
$$

Then $T$ is narrow if and only if $T \tilde{+} x^{*}$ is a strong Daugavet operator for every $x^{*} \in X^{*}$.

A $\Gamma$-version of the definition of a narrow operator will be also useful for us.

Definition 2.3. An operator $T$ on a Banach space $X$ is said to be narrow with respect to a subset $\Gamma \subset S\left(X^{*}\right)(T \in \mathcal{N A \mathcal { R }}(X, \Gamma)$ for short) if for every two elements $x, y \in S(X)$, for every $x^{*} \in \Gamma$ and for every $\varepsilon>0$ there is an element $z \in S(X)$ such that $\|x+z\| \geq 2-\varepsilon$ and $\|T(y-z)\|+\left|x^{*}(y-z)\right| \leq \varepsilon$.

It will be technically convenient to work with the case of $\varepsilon=0$ in the above definitions. Therefore we introduce "rigid versions" of these notions.

\section{Definition 2.4.}

(a) A Banach space $X$ has the rigid Daugavet property with respect to a subset $\Gamma \subset S\left(X^{*}\right)\left(X \in \operatorname{DP}^{r}(\Gamma)\right.$ for short) if for every $x \in S(X)$ and $x^{*} \in \Gamma$ there exists some $y \in S(X)$ such that $x^{*}(y)=1$ and $\|x+y\|=2$.

(b) An operator $T$ on a Banach space $X$ is said to be a rigid strong Daugavet operator (in symbols $T \in \mathcal{S D}^{r}(X)$ ) if for every two elements $x, y \in S(X)$ there is an element $z \in S(X)$ such that $\|x+z\|=2$ and $T(y-z)=0$.

(c) An operator $T$ is said to be rigidly narrow with respect to a subset $\Gamma \subset S\left(X^{*}\right)$ (in symbols $T \in \mathcal{N A R}^{r}(X, \Gamma)$ ) if for every two elements $x, y \in S(X)$ and for every $x^{*} \in \Gamma$ there is an element $z \in S(X)$ such that $\|x+z\|=2$ and $\|T(y-z)\|+\left|x^{*}(y-z)\right|=0$.

Let us mention that a rigid strong Daugavet operator is necessarily noninjective. To see this, one just has to apply the definition for $y=-x$; then $y-z$ will be a nonzero element which $T$ maps to 0 . Using this remark one can easily prove the following statement.

Lemma 2.5. If $T \in \mathcal{S D}^{r}(X)$, then for every $x \in S(X)$ and $y \in B(X)$ there is an element $z \in S(X)$ such that $\|x+z\|=2$ and $T(y-z)=0$.

Proof. Using the non-injectivity of $T$ one can find an element $y_{1} \in S(X)$ such that $T\left(y-y_{1}\right)=0$. Then applying the definition of $\mathcal{S D}^{r}(X)$ to $x$ and $y_{1}$ one obtains an element $z \in S(X)$ such that $\|x+z\|=2$ and $T\left(y_{1}-z\right)=0$. But for this element $T(y-z)=0$, too.

For many investigations in the context of the Daugavet property the study of the rigid notions above turns out to be sufficient, but is technically more feasible. The connection between the original versions and their rigid variants is made using ultrapowers. We refer for instance to [2] for an introduction to ultrapowers of Banach spaces. 
Let $\mathcal{U}$ be a nontrivial ultrafilter on $\mathbb{N}, T$ be an operator acting from a Banach space $X$ to a Banach space $Y, \Gamma \subset S\left(X^{*}\right)$. We denote by $T^{\mathcal{U}}$ the natural operator between the ultrapowers $X^{\mathcal{U}}$ and $Y^{\mathcal{U}}$ defined by $T^{\mathcal{U}}\left(x_{n}\right)=$ $\left(T x_{n}\right)$, and by $\Gamma^{\mathcal{U}}$ we denote the set of the linear functionals $F=\left(f_{n}\right)$, $f_{n} \in \Gamma$, of the form $F\left(x_{n}\right)=\lim _{\mathcal{U}} f_{n}\left(x_{n}\right)$.

\section{Lemma 2.6.}

1. If $X \in \operatorname{DP}(\Gamma)$, then $X^{\mathcal{U}} \in \operatorname{DP}^{r}\left(\Gamma^{\mathcal{U}}\right)$.

2. If $X^{\mathcal{U}} \in \mathrm{DP}\left(\Gamma^{\mathcal{U}}\right)$, then $X \in \operatorname{DP}(\Gamma)$.

3. If $T \in \mathcal{S D}(X)$, then $T^{\mathcal{U}} \in \mathcal{S D}^{r}\left(X^{\mathcal{U}}\right)$.

4. If $T^{\mathcal{U}} \in \mathcal{S D}\left(X^{\mathcal{U}}\right)$, then $T \in \mathcal{S D}(X)$.

5. If $T \in \mathcal{N} \mathcal{N} \mathcal{R}(X, \Gamma)$, then $T^{\mathcal{U}} \in \mathcal{N A R}^{r}\left(X, \Gamma^{\mathcal{U}}\right)$.

6. If $T^{\mathcal{U}} \in \mathcal{N} \mathcal{N A R}\left(X, \Gamma^{\mathcal{U}}\right)$, then $T \in \mathcal{N} \mathcal{N A R}(X, \Gamma)$.

Proof. All these statements don't differ too much in essence. Let us prove for example (5). Fix arbitrary $x=\left(x_{n}\right), y=\left(y_{n}\right) \in S\left(X^{\mathcal{U}}\right)$ and $x^{*}=\left(x_{n}^{*}\right) \in \Gamma^{\mathcal{U}}$. Without loss of generality (just replacing one representation of an element in $X^{\mathcal{U}}$ by another) one may assume that $x_{n}, y_{n} \in S(X)$ for all $n \in \mathbb{N}$. Applying the condition $T \in \mathcal{N A R}(X, \Gamma)$ for $x_{n}, y_{n}, x_{n}^{*}$ and $\varepsilon=\frac{1}{n}$ we obtain elements $z_{n} \in S(X)$ such that $\left\|x_{n}+z_{n}\right\|>2-\frac{1}{n}$ and $\left\|T\left(y_{n}-z_{n}\right)\right\|+\left|x_{n}^{*}\left(y_{n}-z_{n}\right)\right|<\frac{1}{n}$. This means that for $z=\left(z_{n}\right) \in S\left(X^{\mathcal{U}}\right)$ the conditions $\|x+z\|=2$ and $\left\|T^{\mathcal{U}}(y-z)\right\|+\left|x^{*}(y-z)\right|=0$ are fulfilled.

\section{Strong Daugavet and narrow operators in $\ell_{\infty}$-SUmS}

We first fix some notation. If $T$ is an operator defined on $X=X_{1} \oplus_{\infty}$ $X_{2}$, we let $T_{1}$ stand for the restriction of $T$ to $X_{1}$, i.e., $T_{1} x_{1}=T\left(x_{1}, 0\right)$; and likewise $T_{2} x_{2}=T\left(0, x_{2}\right)$ defines the restriction to $X_{2}$. Thus for $x=$ $\left(x_{1}, x_{2}\right) \in X, T x=T\left(x_{1}, x_{2}\right)=T_{1} x_{1}+T_{2} x_{2}$.

The aim of this section is to prove that $T$ is a strong Daugavet operator if and only if both restrictions $T_{1}$ and $T_{2}$ of $T$ are strong Daugavet operators. The same is true for narrow operators.

Proposition 3.1. If $X=X_{1} \oplus_{\infty} X_{2}$ and $T_{i} \in \mathcal{S D}\left(X_{i}\right)\left(T_{i} \in \mathcal{S D}^{r}\left(X_{i}\right)\right.$ ) for $i=1,2$, then $T \in \mathcal{S D}(X)\left(T \in \mathcal{S D}^{r}(X)\right.$ respectively).

Proof. By Lemma 2.6 it is sufficient to consider only the "rigid" version of the proposition. Indeed, we have $X^{\mathcal{U}}=X_{1}^{\mathcal{U}} \oplus_{\infty} X_{2}^{\mathcal{U}}$ and $\left(T^{\mathcal{U}}\right)_{i}=\left(T_{i}\right)^{\mathcal{U}}$. Therefore, if $T_{i} \in \mathcal{S D}\left(X_{i}\right)$, then $\left(T_{i}\right)^{\mathcal{U}} \in \mathcal{S D}^{r}\left(X_{i}^{\mathcal{U}}\right)$ and, assuming the rigid version, we conclude that $T^{\mathcal{U}} \in \mathcal{S D}^{r}\left(X^{\mathcal{U}}\right)$ which implies $T \in \mathcal{S D}(X)$.

Thus, we need to prove that for every $x=\left(x_{1}, x_{2}\right)$ with $\|x\|=\max \left\{\left\|x_{1}\right\|\right.$, $\left.\left\|x_{2}\right\|\right\}=1$ and $y=\left(y_{1}, y_{2}\right)$ with $\|y\|=\max \left\{\left\|y_{1}\right\|,\left\|y_{2}\right\|\right\}=1$, there is some $z=\left(z_{1}, z_{2}\right)$ with $\|z\|=\max \left\{\left\|z_{1}\right\|,\left\|z_{2}\right\|\right\}=1$ such that $\|x+z\|=\max \left\{\| x_{1}+\right.$ $\left.z_{1}\|,\| x_{2}+z_{2} \|\right\}=2$ and $\|T(y-z)\|=\left\|T_{1}\left(y_{1}-z_{1}\right)+T_{2}\left(y_{2}-z_{2}\right)\right\|=0$.

Without any loss of generality we may assume that $\left\|x_{1}\right\|=1$. Using Lemma 2.5 for $T_{1} \in \mathcal{S D}^{r}\left(X_{1}\right)$, we can find, given $x_{1} \in S(X)$ and $y_{1} \in B(X)$, some $z_{1} \in S(X)$ with $\left\|x_{1}+z_{1}\right\|=2$ and $\left\|T_{1}\left(y_{1}-z_{1}\right)\right\|=0$. Put $z_{2}=y_{2}$, 
$z=\left(z_{1}, z_{2}\right)$; then $\|z\|=1$ and $\|x+z\| \geq\left\|x_{1}+z_{1}\right\|=2$ and

$$
\|T(y-z)\|=\left\|T_{1}\left(y_{1}-z_{1}\right)+T_{2}\left(y_{2}-z_{2}\right)\right\|=\left\|T_{1}\left(y_{1}-z_{1}\right)\right\|=0,
$$

completing the proof.

Corollary 3.2. If $X=X_{1} \oplus_{\infty} X_{2}$ and $T_{i} \in \mathcal{N A A R}\left(X_{i}\right)$ for $i=1,2$, then $T \in \mathcal{N A R}(X)$.

Proof. We have to prove that for each $x^{*}=\left(x_{1}^{*}, x_{2}^{*}\right) \in X^{*}=X_{1}^{*} \oplus_{1} X_{2}^{*}$, $T \tilde{+} x^{*}$ is a strong Daugavet operator; see Section 2 for this notation.

Let us consider the restriction of $T \tilde{+} x^{*}$ to $X_{1}$; then

$$
\begin{aligned}
\left\|\left(T \tilde{+} x^{*}\right)_{1} x_{1}\right\| & =\left\|\left(T \tilde{+} x^{*}\right)\left(x_{1}, 0\right)\right\| \\
& =\left\|T\left(x_{1}, 0\right)\right\|+\left|x^{*}\left(\left(x_{1}, 0\right)\right)\right| \\
& =\left\|T_{1} x_{1}\right\|+\left|x_{1}^{*}\left(x_{1}\right)\right| .
\end{aligned}
$$

Since $T_{1}$ is narrow, $T_{1} \tilde{+} x_{1}^{*}$ is a strong Daugavet operator and hence so is $\left(T \tilde{+} x^{*}\right)_{1}$. By symmetry, the same is true for the restriction to $X_{2}$, and Proposition 3.1 implies that $T \tilde{+} x^{*}$ is a strong Daugavet operator. Since $x^{*}$ is arbitrary, $T$ is narrow.

We now turn to the converse of Proposition 3.1. In the sequel we shall call elements $x_{1}, \ldots, x_{n}$ of a normed space quasi-collinear if

$$
\left\|x_{1}+\cdots+x_{n}\right\|=\left\|x_{1}\right\|+\cdots+\left\|x_{n}\right\| .
$$

We will need a simple lemma.

Lemma 3.3. Suppose that $x_{1}, \ldots, x_{n}$ are quasi-collinear.

(a) $\left\|a_{1} x_{1}+\cdots+a_{n} x_{n}\right\|=a_{1}\left\|x_{1}\right\|+\cdots+a_{n}\left\|x_{n}\right\|$ for all nonnegative coefficients $a_{k}$.

(b) If $x_{n+1}$ is quasi-collinear to $\left(x_{1}+\cdots+x_{n}\right) / n$, then all the vectors $x_{1}, \ldots, x_{n+1}$ are quasi-collinear.

Proof. (a) The function $F: \mathbb{R}_{+}^{n} \rightarrow \mathbb{R}$ defined by

$$
F\left(a_{1}, \ldots, a_{n}\right)=\left\|a_{1} x_{1}+\cdots+a_{n} x_{n}\right\|-\left(a_{1}\left\|x_{1}\right\|+\cdots+a_{n}\left\|x_{n}\right\|\right)
$$

is convex, takes values $\leq 0$ and $F(1, \ldots, 1)=0$. Hence $F=0$.

(b) follows from (a):

$$
\begin{aligned}
\left\|x_{1}+\cdots+x_{n}+x_{n+1}\right\| & =\left\|n \frac{x_{1}+\cdots+x_{n}}{n}+x_{n+1}\right\| \\
& =n\left\|\frac{x_{1}+\cdots+x_{n}}{n}\right\|+\left\|x_{n+1}\right\| \\
& =\left\|x_{1}\right\|+\cdots+\left\|x_{n}\right\|+\left\|x_{n+1}\right\| .
\end{aligned}
$$

Theorem 3.4. If $X=X_{1} \oplus_{\infty} X_{2}$, then for every strong Daugavet operator $T$ on $X$ the restrictions $T_{1}$ and $T_{2}$ of $T$ to $X_{1}$ and $X_{2}$ are strong Daugavet operators. 
Proof. As in Proposition 3.1 it is sufficient to prove that $T_{1} \in \mathcal{S D}\left(X_{1}\right)$ whenever $T \in \mathcal{S D}^{r}(X)$.

So let $T \in \mathcal{S D}^{r}(X), x_{1}, y_{1} \in S\left(X_{1}\right)$ and $\varepsilon>0$. Apply the definition of a rigid strong Daugavet operator to $x=\left(x_{1}, 0\right), y=\left(y_{1}, 0\right)$. We get some $z^{1}=\left(z_{1}^{1}, z_{2}^{1}\right)$ for which $\left\|y_{1}+z_{1}^{1}\right\|=1,\left\|z_{2}^{1}\right\| \leq 1,\left\|x_{1}+y_{1}+z_{1}^{1}\right\|=2$ and $T z^{1}=0$. This means, in particular, that the vectors $x_{1}$ and $y_{1}+z_{1}^{1}$ are quasicollinear. Now apply the definition of a rigid strong Daugavet operator to $x=\left(\left(x_{1}+y_{1}+z_{1}^{1}\right) / 2,0\right), y=\left(y_{1}, z_{2}^{1}\right)$. We get some $z^{2}=\left(z_{1}^{2}, z_{2}^{2}\right)$ for which $T z^{2}=0,\left\|y_{1}+z_{1}^{2}\right\|=1,\left\|z_{2}^{1}+z_{2}^{2}\right\| \leq 1$ and $\left\|\left(x_{1}+y_{1}+z_{1}^{1}\right) / 2+\left(y_{1}+z_{1}^{2}\right)\right\|=2$. This again means, by Lemma 3.3, that the vectors $x_{1}, y_{1}+z_{1}^{1}$ and $y_{1}+z_{1}^{2}$ are quasi-collinear. Now apply the same token to $x=\left(\left(x_{1}+\left(y_{1}+z_{1}^{1}\right)+\right.\right.$ $\left.\left.\left(y_{1}+z_{1}^{2}\right)\right) / 3,0\right)$ and $y=\left(y_{1}, z_{2}^{1}+z_{2}^{2}\right)$, etc.

Continuing this process we obtain a sequence $z^{n}=\left(z_{1}^{n}, z_{2}^{n}\right)$ for which all the vectors $x_{1}, y_{1}+z_{1}^{1}, y_{1}+z_{1}^{2}, \ldots$ are quasi-collinear unit vectors, $\| z_{2}^{1}+$ $\cdots+z_{2}^{n} \| \leq 1$ and $T z^{n}=0$. Consider $z=\left(z_{1}^{1}+z_{1}^{2}+\cdots+z_{1}^{n}\right) / n \in X_{1}$. By construction and Lemma $3.3\left\|x_{1}+y_{1}+z\right\|=2,\left\|y_{1}+z\right\|=1$ and

$$
\left\|T_{1} z\right\|=\|T(z, 0)\|=\left\|T\left(0,\left(z_{2}^{1}+z_{2}^{2}+\cdots+z_{2}^{n}\right) / n\right)\right\| \leq\|T\| / n .
$$

Because $n$ can be taken arbitrarily big, this proves that $T_{1} \in \mathcal{S D}\left(X_{1}\right)$.

Corollary 3.5. If $X=X_{1} \oplus_{\infty} X_{2}$, then for every narrow operator $T$ on $X$ the restrictions $T_{1}$ and $T_{2}$ of $T$ to $X_{1}$ and $X_{2}$ are narrow operators.

Proof. This follows directly from Theorem 3.4 and the definition of a narrow operator.

Let $X_{1}$ be an $M$-ideal of a Banach space $X$ (see [4] for a study of $M$ ideals) and $T$ be a strong Daugavet operator on $X$. We haven't been able to decide whether the restriction of $T$ to $X_{1}$ is a strong Daugavet operator again. This would give us the operator version of the result saying that an $M$-ideal in a space with the Daugavet property has the Daugavet property itself [5, Prop 2.10].

\section{Strong Daugavet and narrow operators in $\ell_{1}$-Sums}

We use the same notation concerning restrictions of operators as before, but for an $\ell_{1}$-sum $X=X_{1} \oplus_{1} X_{2}$.

Proposition 4.1. If $X=X_{1} \oplus_{1} X_{2}$ and $T_{i} \in \mathcal{S D}\left(X_{i}\right)\left(T_{i} \in \mathcal{S D}^{r}\left(X_{i}\right)\right.$ ) for $i=1,2$, then $T \in \mathcal{S D}(X)\left(T \in \mathcal{S D}^{r}(X)\right.$ respectively).

Proof. Again, by Lemma 2.6 it is sufficient to consider only the "rigid" version of the theorem. Thus, we need to prove that for every $x=\left(x_{1}, x_{2}\right)$ with $\|x\|=\left\|x_{1}\right\|+\left\|x_{2}\right\|=1$ and $y=\left(y_{1}, y_{2}\right)$ with $\|y\|=\left\|y_{1}\right\|+\left\|y_{2}\right\|=1$, there is some $z=\left(z_{1}, z_{2}\right)$ with $\|z\|=\left\|z_{1}\right\|+\left\|z_{2}\right\|=1$ such that $\|x+z\|=$ $\left\|x_{1}+z_{1}\right\|+\left\|x_{2}+z_{2}\right\|=2$ and $\|T(y-z)\|=\left\|T_{1}\left(y_{1}-z_{1}\right)+T_{2}\left(y_{2}-z_{2}\right)\right\|=0$. 
For $i=1,2$, since $T_{i} \in \mathcal{S D}^{r}\left(X_{i}\right)$, we can produce, using Lemma 2.5, some $z_{i} \in\left\|y_{i}\right\| S\left(X_{i}\right)$ with $\left\|x_{i}+z_{i}\right\|=\left\|x_{i}\right\|+\left\|z_{i}\right\|$ and $\left\|T_{i}\left(y_{i}-z_{i}\right)\right\|=0$. Now let us take $z=\left(z_{1}, z_{2}\right)$; then

$$
\begin{aligned}
\|z\|=\left\|z_{1}\right\|+\left\|z_{2}\right\| & =\left\|y_{1}\right\|+\left\|y_{2}\right\|=1 \\
\|x+z\|=\left\|x_{1}+z_{1}\right\|+\left\|x_{2}+z_{2}\right\| & =\left\|x_{1}\right\|+\left\|z_{1}\right\|+\left\|x_{2}\right\|+\left\|z_{2}\right\|=2
\end{aligned}
$$

and

$$
T(y-z)=T_{1}\left(y_{1}-z_{1}\right)+T_{2}\left(y_{2}-z_{2}\right)=0 .
$$

So, $z$ satisfies all the conditions above, and the proposition is proved.

By the same argument as in Corollary 3.2 we obtain:

Corollary 4.2. If $X=X_{1} \oplus_{1} X_{2}$ and $T_{i} \in \mathcal{N A R}\left(X_{i}\right)$ for $i=1,2$, then $T \in \mathcal{N A R}(X)$.

We now study the converse of these results. Let us recall that a subset $\Gamma \subset S\left(X^{*}\right)$ is said to be 1-norming if

$$
\|x\|=\sup _{x^{*} \in \Gamma} x^{*}(x) .
$$

for every $x \in X$. A subset $\Gamma \subset S\left(X^{*}\right)$ is said to be a boundary for $X$ if the above supremum is always attained, i.e., if for every $x \in X$ there is some $x^{*} \in \Gamma$ such that $x^{*}(x)=\|x\|$. Clearly, the notion of a boundary is a "rigid" version of a 1-norming set. It is easy to check that $\Gamma^{\mathcal{U}}$ is a boundary for $X^{\mathcal{U}}$ if and only if $\Gamma$ is 1-norming.

Lemma 4.3. Let $X=X_{1} \oplus_{1} X_{2}$, let $\Gamma_{j} \subset S\left(X_{j}^{*}\right)$ be boundaries for $X_{j}$ for $j=1,2$, and let $\Gamma=\Gamma_{1} \cup \Gamma_{2}$. If $T \in \mathcal{N A R}^{r}(X, \Gamma)$, then $T_{j}$, the restrictions of $T$ to $X_{j}$, are rigid strong Daugavet operators.

Proof. Let us consider the case of $T_{1}$. We have to prove that for every $x_{1}, y_{1} \in S\left(X_{1}\right)$ there exists some $u_{1} \in S\left(X_{1}\right)$ such that $\left\|x_{1}+u_{1}\right\|=2$ and $T_{1}\left(u_{1}-y_{1}\right)=0$.

Let us take $x=\left(x_{1}, 0\right), y=\left(y_{1}, 0\right) \in S(X)$ and a functional $x_{1}^{*} \in \Gamma_{1}$ such that $x_{1}^{*}\left(y_{1}\right)=1$. Let us further take $x^{*}=\left(x_{1}^{*}, 0\right) \in \Gamma$. Since $T$ is narrow, we can apply Definition 2.4 with the elements $x, y$ and $x^{*}$ defined above; thus, there exists some $z=\left(z_{1}, z_{2}\right) \in S(X)$ such that

$$
\|x+z\|=\left\|x_{1}+z_{1}\right\|+\left\|z_{2}\right\|=2
$$

and

$$
\|T(z-y)\|+\left|x^{*}(z-y)\right|=\|T(z-y)\|+\left|x_{1}^{*}\left(z_{1}-y_{1}\right)\right|=0 .
$$

From the last condition we obtain $\left|x_{1}^{*}\left(z_{1}-y_{1}\right)\right|=0$. Keeping in mind that $x_{1}^{*}\left(y_{1}\right)=1$, we get $x_{1}^{*}\left(z_{1}\right)=1$. But $\left\|x_{1}^{*}\right\|=1$, so $\left\|z_{1}\right\|=1$. Then

$$
\left\|z_{2}\right\|=0,
$$

because $\left\|z_{1}\right\|+\left\|z_{2}\right\|=1$. So $\left\|x_{1}+z_{1}\right\|=\|x+z\|=2$ and by (4.1) and (4.2) $T_{1}\left(y_{1}\right)=T(y)=T(z)=T_{1}\left(z_{1}\right)$. Thus the definition of a rigid strong Daugavet operator is fulfilled for $T_{1}$. 
We can now prove the converse of Corollary 4.2 .

Theorem 4.4. Let $X=X_{1} \oplus_{1} X_{2}$ and $T \in \mathcal{N A R}(X)$. Then $T_{1}$ and $T_{2}$, the restrictions of $T$ to $X_{1}$ and $X_{2}$, are narrow operators.

Proof. It has been proved in [6, Cor. 3.14] that if $T$ is narrow then so is $T \tilde{+} x^{*}$ for any $x^{*} \in X^{*}$, in particular for $x^{*} \in \Gamma=X_{1}^{*} \cup X_{2}^{*}$. By Lemma 2.6 we may pass to ultraproducts, apply the previous lemma, pass back to the original space and obtain that $T_{1} \tilde{+} x_{1}^{*}$ is strongly Daugavet for every $x_{1}^{*} \in X_{1}^{*}$. Hence, by definition, $T_{1}$ is narrow, and by symmetry, so is $T_{2}$.

However, the analogue of Theorem 4.4 for strong Daugavet operators, i.e., the converse of Proposition 4.1, is false.

Proposition 4.5. Let $X=X_{1} \oplus_{1} X_{2}$ and $T \in \mathcal{S D}(X)$. Then $T_{1}$, the restriction of $T$ to $X_{1}$, need not be a strong Daugavet operator.

Proof. The sum functional $T x=\sum_{n=1}^{\infty} x(n)$ is a strong Daugavet operator on $\ell_{1}=\mathbb{R} \oplus_{1} X_{2}$ (see [3, Prop. 2.4]), yet its restriction to $\mathbb{R}$ (i.e., the span of $\left.e_{1}\right)$ is not.

We wish to indicate another counterexample that even works on a space with the Daugavet property, viz. $L_{1}[0,1]$. For this, let us recall the main features of the example from Theorem 6.3 of [6]. In this example subspaces $Y_{1} \subset L_{1}[0,1]$ and $Y=Y_{1} \oplus \operatorname{lin}\{1\}$ and a measurable subset $P \subset[0,1]$ of measure $\mu(P)<1 / 9$ with the following properties are constructed:

$$
\left\|g \chi_{[0,1] \backslash P}\right\| \leq 3\left\|g \chi_{P}\right\| \quad \forall g \in Y_{1}
$$

and the quotient map $q: L_{1}[0,1] \rightarrow L_{1}[0,1] / Y$ is a strong Daugavet operator.

Now let $Q \subset[0,1], \mu(Q)<1 / 3, Q \cap P=\emptyset$. Then the restriction of $q$ to $L_{1}(Q)$ is bounded from below. So in particular this restriction is not a strong Daugavet operator; observe that $L_{1}[0,1]=L_{1}(Q) \oplus_{1} L_{1}([0,1] \backslash Q)$.

Indeed, let us assume to the contrary that the restriction of $q$ to $L_{1}(Q)$ is unbounded from below. This means that for every $\varepsilon>0$ there exists a function $f \in L_{1}(Q)$, a function $g_{1} \in Y_{1}$ and a constant $a$ such that

$$
\left\|f-\left(g_{1}+a\right)\right\|<\varepsilon \text {. }
$$

Denote $[0,1] \backslash(P \cup Q)$ by $S$; then $\mu(S)>1 / 2$. Then $\left\|\left(a+g_{1}\right) \chi_{P \cup S}\right\|<\varepsilon$ and

$$
\begin{aligned}
a \mu(P)=\left\|a \chi_{P}\right\| & \geq\left\|g_{1} \chi_{P}\right\|-\varepsilon \geq \frac{1}{3}\left\|g_{1} \chi_{S}\right\|-\varepsilon \quad \text { (by 4.3) } \\
& \geq \frac{1}{3}\left\|a \chi_{S}\right\|-2 \varepsilon=\frac{1}{3} a \mu(S)-2 \varepsilon,
\end{aligned}
$$

so $a<40 \varepsilon$. This means that $\left\|f-g_{1}\right\|<41 \varepsilon$. On the other hand

$$
\begin{aligned}
\left\|f-g_{1}\right\| & \geq\left\|\left(f-g_{1}\right) \chi_{P}\right\|=\left\|g_{1} \chi_{P}\right\| \\
& \geq \frac{1}{3}\left\|g_{1} \chi_{Q}\right\| \geq \frac{1}{3}\left(\|f\|-\left\|\left(f-g_{1}\right) \chi_{Q}\right\|\right) \geq \frac{1}{3}(1-41 \varepsilon),
\end{aligned}
$$

which is a contradiction. 


\section{The Daugavet property for unConditional sums of SPACES}

Throughout this section $F$ denotes a Banach space with a 1-unconditional normalised Schauder basis. We can think of the elements of $F$ as sequences with the property that

$$
\left\|\left(a_{1}, a_{2}, \ldots\right)\right\|_{F}=\left\|\left(\left|a_{1}\right|,\left|a_{2}\right|, \ldots\right)\right\|_{F} \quad \forall\left(a_{j}\right) \in F .
$$

Note that $F$ is naturally endowed with the structure of a Banach lattice with respect to the pointwise operations.

Suppose that $X_{1}, X_{2}, \ldots$ are Banach spaces. Their $F$-sum $X=\left(X_{1}\right.$, $\left.X_{2}, \ldots\right)_{F}$ consists of all sequences $\left(x_{j}\right)$ with $x_{j} \in X_{j}$ and $\left(\left\|x_{j}\right\|\right) \in F$ with the norm $\left\|\left(x_{j}\right)\right\|=\left\|\left(\left\|x_{j}\right\|\right)\right\|_{F}$. We are going to characterise when such an $F$-sum has the Daugavet property.

Theorem 5.1. Let $X_{1}, X_{2}, \ldots$ be Banach spaces with the Daugavet property. Then their F-sum $X$ has the Daugavet property if and only if the Banach lattice $F$ has the positive Daugavet property in the sense that $\|\mathrm{Id}+T\|=$ $1+\|T\|$ whenever $T: F \rightarrow F$ is a positive rank-1 operator.

Proof. We first remark that the positive Daugavet property may be characterised as in Lemma 2.1; the proof is verbatim the same as in [5, Lemma 2.1].

Lemma 5.2. A Banach lattice has the positive Daugavet property if and only if for every positive $a \in S(F)$, every positive $a^{*} \in S\left(F^{*}\right)$ and every $\varepsilon>0$ there is some positive $b \in S(F)$ such that $a^{*}(b) \geq 1-\varepsilon$ and $\|a+b\| \geq 2-\varepsilon$.

Now suppose that $X$ has the Daugavet property; we shall verify the condition of Lemma 5.2. Note that $F^{*}$ can be represented by all sequences $\left(a_{j}^{*}\right)$ such that

$$
\sup _{n}\left\|\left(\left|a_{1}^{*}\right|, \ldots,\left|a_{n}^{*}\right|, 0,0, \ldots\right)\right\|_{F^{*}}<\infty,
$$

and $X^{*}$ can be represented by all sequences $\left(x_{j}^{*}\right), x_{j}^{*} \in X_{j}^{*}$, such that

$$
\left\|x^{*}\right\|=\sup _{n}\left\|\left(\left\|x_{1}^{*}\right\|, \ldots,\left\|x_{n}^{*}\right\|, 0,0, \ldots\right)\right\|_{F^{*}}<\infty .
$$

Let $a=\left(a_{j}\right) \in S(F)$ and $a^{*}=\left(a_{j}^{*}\right) \in S\left(F^{*}\right)$ be positive elements and let $\varepsilon>0$. Pick $x_{j} \in X_{j}$ and $x_{j}^{*} \in X_{j}^{*}$ such that $\left\|x_{j}\right\|=a_{j},\left\|x_{j}^{*}\right\|=a_{j}^{*}$ and put $x=\left(x_{j}\right), x^{*}=\left(x_{j}^{*}\right)$; then $\|x\|=\left\|x^{*}\right\|=1$. Since $X$ has the Daugavet property, we can find $y \in S(X)$ such that $x^{*}(y) \geq 1-\varepsilon$ and $\|x+y\| \geq 2-\varepsilon$; cf. Lemma 2.1. Write $y=\left(y_{j}\right)$ and $b=\left(\left\|y_{j}\right\|\right)$; then $\|b\|_{F}=1$ and

$$
\begin{gathered}
1-\varepsilon \leq x^{*}(y)=\sum_{j=1}^{\infty} x_{j}^{*}\left(y_{j}\right) \leq \sum_{j=1}^{\infty}\left\|x_{j}^{*}\right\|\left\|y_{j}\right\|=a^{*}(b), \\
2-\varepsilon \leq\|x+y\|=\left\|\left(\left\|x_{j}+y_{j}\right\|\right)\right\|_{F} \leq\left\|\left(\left\|x_{j}\right\|+\left\|y_{j}\right\|\right)\right\|_{F} \leq\|a\|+\|b\|,
\end{gathered}
$$

where we have used the fact that the norm of $F$ is monotonic in each variable. Hence $F$ has the positive Daugavet property. (Incidentally, the assumption that the $X_{j}$ have the Daugavet property did not enter this part of the proof.) 
Conversely, suppose that $F$ has the positive Daugavet property. Let $x=$ $\left(x_{j}\right) \in S(X)$ and $x^{*}=\left(x_{j}^{*}\right) \in S\left(X^{*}\right)$, define $a=\left(a_{j}\right)=\left(\left\|x_{j}\right\|\right) \in S(F)$ and $a^{*}=\left(a_{j}^{*}\right)=\left(\left\|x_{j}^{*}\right\|\right) \in S\left(F^{*}\right)$. Given $\varepsilon>0$, find using Lemma 5.2 some $b=\left(b_{j}\right) \in S(F)$ such that $a^{*}(b) \geq 1-\varepsilon$ and $\|a+b\| \geq 2-\varepsilon$. Since $X_{j}$ has the Daugavet property, one can find $y_{j} \in X_{j}$ such that $\left\|y_{j}\right\|=$ $b_{j}, x_{j}^{*}\left(y_{j}\right) \geq(1-\varepsilon) a_{j}^{*} b_{j}$ and $\left\|x_{j}+y_{j}\right\| \geq(1-\varepsilon)\left(a_{j}+b_{j}\right)$; just note that $\left\|\mathrm{Id}+\left(x_{j}^{*} / a_{j}^{*}\right) \otimes\left(x_{j} / b_{j}\right)\right\|=1+a_{j} / b_{j}$. Therefore $y=\left(y_{j}\right) \in S(X)$ satisfies

$$
x^{*}(y)=\sum_{j=1}^{\infty} x_{j}^{*}\left(y_{j}\right) \geq(1-\varepsilon) \sum_{j=1}^{\infty} a_{j}^{*} b_{j}=(1-\varepsilon) a^{*}(b) \geq(1-\varepsilon)^{2}
$$

and

$$
\begin{aligned}
\|x+y\| & =\left\|\left(\left\|x_{j}+y_{j}\right\|\right)\right\|_{F} \geq(1-\varepsilon)\left\|\left(\left\|x_{j}\right\|+\left\|y_{j}\right\|\right)\right\|_{F} \\
& =(1-\varepsilon)\|a+b\|_{F} \geq 2(1-\varepsilon)(1-2 \varepsilon) .
\end{aligned}
$$

Hence $X$ has the Daugavet property.

It is clear that for example $c_{0}$ and $\ell_{1}$ have the positive Daugavet property, hence Theorem 5.1 contains [5, Prop. 2.16] as a special case. Also, if $F^{*}$ is a Banach lattice with the positive Daugavet property, then so is $F$.

If $F$ is finite-dimensional, we can pass to the limit $\varepsilon=0$ in Lemma 5.2 by compactness. Thus, we obtain the following variant of Theorem 5.1.

Corollary 5.3. Let $\operatorname{dim} F=n$ and $X_{1}, \ldots, X_{n}$ be Banach spaces with the Daugavet property. Then their F-sum $\left(X_{1} \oplus \cdots \oplus X_{n}\right)_{F}$ has the Daugavet property if and only if for every positive $a \in S(F)$ and every positive $a^{*} \in$ $S\left(F^{*}\right)$ there is some $b \in S(F)$ such that $a^{*}(b)=1$ and $\|a+b\|=2$.

This condition can be rephrased geometrically as follows. For any point $a \geq 0$ in $S(F)$ and any supporting hyperplane of the positive part of the unit sphere $H=\left\{a^{*}=1\right\}$ there is a line segment in the unit sphere that contains $a$ and intersects $H \cap S(F)$. From this the following corollary is evident.

Corollary 5.4. If $X=\left(X_{1} \oplus X_{2}\right)_{F}$ has the Daugavet property, then either $F=\ell_{1}^{2}$ or $F=\ell_{\infty}^{2}$, i.e., either $X=X_{1} \oplus_{1} X_{2}$ or $X=X_{1} \oplus_{\infty} X_{2}$.

It is easy to see that $F_{1} \oplus_{1} F_{2}$ and $F_{1} \oplus_{\infty} F_{2}$ have the positive Daugavet property whenever $F_{1}$ and $F_{2}$ have; in fact, the proof of Theorem 5.1 shows that the $F$-sum $\left(F_{1} \oplus F_{2} \oplus \ldots\right)_{F}$ of Banach lattices with the positive Daugavet property is a Banach lattice with the positive Daugavet property. Therefore, starting from the real line we can form $\ell_{1}$-sums and $\ell_{\infty}$-sums consecutively to obtain finite-dimensional spaces with the positive Daugavet property, e.g., the 18-dimensional space

$$
\left(\ell_{\infty}^{3} \oplus_{1} \ell_{\infty}^{4}\right) \oplus_{\infty}\left(\ell_{1}^{3} \oplus_{1} \ell_{\infty}^{3}\right) \oplus_{\infty} \ell_{1}^{5} .
$$

However, there are other examples, even in the three-dimensional case; for

$$
\left\|\left(a_{1}, a_{2}, a_{3}\right)\right\|_{F}=\max \left\{\left|a_{1}\right|+\frac{\left|a_{3}\right|}{2},\left|a_{2}\right|+\left|a_{3}\right|\right\}
$$


defines a norm on $\mathbb{R}^{3}$ with the positive Daugavet property. In this example the unit sphere intersected with the half-space $\{(0,0, t): t \geq 0\}$ looks like a hip roof and the positive part of $B(F)$, i.e., $B(F) \cap \mathbb{R}_{+}^{3}$, is the convex hull of the points $(0,0,0),(1,0,0),(0,1,0),(0,0,1),(1,1,0)$ and $(1 / 2,0,1)$. From this description it is easy to see (literally) that this norm has the positive Daugavet property.

\section{The Daugavet Property For Ultraproducts}

Let $Z$ be a subspace in $X^{*}, \Gamma=S(Z)$ be a boundary for $X$, and $X \in$ $\operatorname{DP}(\Gamma)$. Is it true that under this condition $X$ has the Daugavet property? Provided the answer to this question is positive Lemma 2.6 easily implies that an ultrapower of a space with the Daugavet property has the Daugavet property itself. Unfortunately we don't know the answer; that is why we investigate the question from another point of view in this section. Note, however, that it is easy to find a Banach space without the Daugavet property that has the Daugavet property with respect to some boundary, e.g., $\ell_{1}$ with the boundary ex $B_{\ell_{1}^{*}}$.

For an element $x \in S(X)$ and $\varepsilon>0$ denote

$$
l^{+}(x, \varepsilon)=\{y \in X:\|y\| \leq 1+\varepsilon,\|x+y\|>2-\varepsilon\} .
$$

The next lemma follows directly from Lemma 2.1.

Lemma 6.1. The following assertions are equivalent:

1. $X$ has the Daugavet property.

2. For every $x \in S(X)$ and every $\varepsilon>0$ the closure of $\operatorname{conv}\left(l^{+}(x, \varepsilon)\right)$ contains $B(X)$.

Lemma 6.1 suggests the following quantitative approach to the Daugavet property. For a subset $A \subset X$ denote by $\operatorname{conv}_{n}(A)$ the set of all convex combinations of all $n$-point collections of elements of $A$. Clearly $\operatorname{conv}(A)=$ $\bigcup_{n \in \mathbb{N}} \operatorname{conv}_{n}(A)$. Denote

$$
\operatorname{Daug}_{n}(X, \varepsilon)=\sup _{x, y \in S(X)} \operatorname{dist}\left(y, \operatorname{conv}_{n}\left(l^{+}(x, \varepsilon)\right)\right)
$$

It is easy to see that for every $\varepsilon>0$ the sequence $\left(\operatorname{Daug}_{n}(X, \varepsilon)\right)$ decreases. If $\lim _{n \rightarrow \infty} \operatorname{Daug}_{n}(X, \varepsilon)=0$ for every $\varepsilon>0$, then $X$ has the Daugavet property. We don't know if the converse is true.

Theorem 6.2. Let $\mathcal{U}$ be an ultrafilter defined on a set $\mathcal{I}$, and let $X_{i}, i \in \mathcal{I}$, be a collection of Banach spaces and $X$ be the corresponding ultraproduct of the $X_{i}$. Then the following assertions are equivalent:

1. $X$ has the Daugavet property.

2. For every $\varepsilon>0, \lim _{\mathcal{U}, n} \operatorname{Daug}_{n}\left(X_{i}, \varepsilon\right)=0$. In other words, for every fixed $\varepsilon>0$ and every $\delta>0$ there is an $n \in \mathbb{N}$ such that the set of all $i$ for which $\operatorname{Daug}_{n}\left(X_{i}, \varepsilon\right)<\delta$ belongs to the ultrafilter $\mathcal{U}$. 
Proof. To deduce (11) from (21) one just has to notice that if the set of all $i$ for which $\operatorname{Daug}_{n}\left(X_{i}, \varepsilon\right)<\delta$ belongs to the ultrafilter $\mathcal{U}$, then $\operatorname{Daug}_{n}(X, \varepsilon)<\delta$. So for every $\varepsilon>0, \operatorname{Daug}_{n}(X, \varepsilon)$ tends to 0 when $n$ tends to infinity, which proves the Daugavet property for $X$.

To deduce (2) from (11) let us argue ad absurdum. Suppose there are $\varepsilon>0$ and $\delta>0$ such that for every $n \in \mathbb{N}$ the set $A_{n}=\left\{i \in \mathcal{I}: \operatorname{Daug}_{n}\left(X_{i}, \varepsilon\right)>\delta\right\}$ belongs to the ultrafilter $\mathcal{U}$. Denote $A_{0}=\mathcal{I}$. Let us construct two elements $x=\left(x_{i}\right)_{i \in \mathcal{I}}$ and $y=\left(y_{i}\right)_{i \in \mathcal{I}}$ of $S(X)$ in such a way that $\left.x_{i}, y_{i} \in S\left(X_{i}\right)\right)$ and for every $i \in A_{n} \backslash A_{n-1}$ the distance from $\operatorname{conv}_{n}\left(l^{+}\left(x_{i}, \varepsilon\right)\right)$ to $y_{i}$ is bigger than $\delta$. The $\operatorname{conv}_{n}$-hull of a set is increasing when $n$ is increasing, so for every $n \in \mathbb{N}$ and every $i \in A_{n}=\bigcup_{m=n}^{\infty} A_{m} \backslash A_{m-1}$ the distance from $\operatorname{conv}_{n}\left(l^{+}\left(x_{i}, \varepsilon\right)\right)$ to $y_{i}$ is bigger than $\delta$. This means in turn that for every $n \in \mathbb{N}, \operatorname{dist}\left(y, \operatorname{conv}_{n}\left(l^{+}(x, \varepsilon)\right)\right) \geq \delta$, so $\operatorname{dist}\left(y, \operatorname{conv}\left(l^{+}(x, \varepsilon)\right)\right) \geq \delta$, which contradicts the Daugavet property of $X$.

Remark 6.3. If $\lim _{n \rightarrow \infty} \operatorname{Daug}_{n}(X, \varepsilon)=0$ for every $\varepsilon>0$, then for every $\varepsilon>0$ there is some $n \in \mathbb{N}$ such that $\operatorname{Daug}_{n}(X, \varepsilon)=0$. More explicitly: If $\operatorname{Daug}_{n}(X, \varepsilon / 2)<\varepsilon / 2$, then $\operatorname{Daug}_{n}(X, \varepsilon)=0$. Moreover for every pair $x, y \in S(X)$ not just $\operatorname{dist}\left(y, \operatorname{conv}_{n}\left(l^{+}(x, \varepsilon)\right)\right)=0$, but $y \in \operatorname{conv}_{n}\left(l^{+}(x, \varepsilon)\right)$.

Proof. Suppose $\operatorname{Daug}_{n}(X, \varepsilon / 2)<\varepsilon / 2$. Fix $x, y \in S(X)$. There exist $y_{1}, \ldots$, $y_{n} \in(1+\varepsilon / 2) B(X),\left\|x+y_{n}\right\|>2-\varepsilon / 2$, and $a_{1}, \ldots, a_{n} \geq 0, \sum_{k=1}^{n} a_{k}=1$, for which $\left\|y-\sum_{k=1}^{n} a_{k} y_{k}\right\|<\varepsilon / 2$. Define elements $z_{j}=y_{j}+y-\sum_{k=1}^{n} a_{k} y_{k}$. Then $z_{j} \in l^{+}(x, \varepsilon), \sum_{j=1}^{n} a_{j} z_{j}=y$, so $y \in \operatorname{conv}_{n}\left(l^{+}(x, \varepsilon)\right)$.

So instead of $\operatorname{Daug}_{n}(X, \varepsilon)$ it is reasonable to consider the following notion, which seems to be a bit more convenient (at least it depends only on one parameter):

$$
D_{X}(\varepsilon)=\inf \left\{n: \operatorname{conv}_{n}\left(l^{+}(x, \varepsilon)\right) \supset S(X) \forall x \in S(X)\right\}
$$

If $D_{X}(\varepsilon)$ is finite for every $\varepsilon>0$, we say that $X$ possesses the uniform Daugavet property. Equivalently, by Remark 6.3, $X$ has the uniform Daugavet property if and only if $\operatorname{Daug}_{n}(X, \varepsilon) \rightarrow 0$ for every $\varepsilon>0$.

The theorem on ultraproducts can be reformulated in the following way.

Theorem 6.4. Let $\mathcal{U}$ be an ultrafilter defined on a set $\mathcal{I}, X_{i}$ be a collection of Banach spaces and $X$ be the corresponding ultraproduct of the $X_{i}$. Then the following assertions are equivalent:

1. $X$ has the Daugavet property.

2. For every $\varepsilon>0$ there exists some $n$ such that the set of all $i$ for which $D_{X_{i}}(\varepsilon)<n$ belongs to the ultrafilter $\mathcal{U}$.

Corollary 6.5. A Banach space $X$ has the uniform Daugavet property if and only if every ultrapower $X^{\mathcal{U}}$ has the Daugavet property, in which case $X^{\mathcal{U}}$ even has the uniform Daugavet property. 
It follows from this corollary and the canonical isometric isomorphism $\left(X \oplus_{\infty} Y\right)^{\mathcal{U}}=X^{\mathcal{U}} \oplus_{\infty} Y^{\mathcal{U}}$ that the uniform Daugavet property is stable by taking $\ell_{\infty}$-direct sums and likewise by taking $\ell_{1}$-direct sums.

Let us prove that the basic examples of spaces with the Daugavet property in fact are spaces with the uniform Daugavet property.

Lemma 6.6. Let $X=L_{1}[0,1]$. If $n>2 / \varepsilon$, then $\operatorname{Daug}_{n}(X, \varepsilon)=0$; if $n \leq 2 / \varepsilon$, then $\operatorname{Daug}_{n}(X, \varepsilon) \leq 1-\varepsilon n /(2+\varepsilon)$. Hence $D_{X}(\varepsilon)$ is of order $\varepsilon^{-1}$.

Proof. Suppose $n>2 / \varepsilon$ and let us take arbitrary points $x$ and $y$ from $S(X)$. There is a partitioning of $[0,1]$ into sets $E_{1}, \ldots, E_{n}$ such that $\left\|x \cdot \chi_{E_{i}}\right\|=$ $1 / n<\varepsilon / 2$. Define functions $y_{i}$ by $y_{i}=\frac{1}{\left\|y \cdot \chi_{E_{i}}\right\|} y \cdot \chi_{E_{i}}$ if $\left\|y \cdot \chi_{E_{i}}\right\| \neq 0$, and $y_{i}=0$ if $\left\|y \cdot \chi_{E_{i}}\right\|=0$. Then $\sum_{i=1}^{n} y_{i} \lambda_{i}=y$, where $\lambda_{i}=\left\|y \cdot \chi_{E_{i}}\right\|$. On the other hand, if $y_{i} \neq 0$, then

$$
\left\|x+y_{i}\right\| \geq\left\|x \cdot \chi_{[0,1] \backslash E_{i}}\right\|+\left\|y_{i}\right\|-\left\|x \cdot \chi_{E_{i}}\right\| \geq 2-2\left\|x \cdot \chi_{E_{i}}\right\|>2-\varepsilon .
$$

So, $y_{i} \in l^{+}(x, \varepsilon)$.

If $n \leq 2 / \varepsilon$, then proceeding as above, with $N=[2 / \varepsilon]+1$ we get a decomposition $E_{1}, \ldots, E_{N}$. Let us arrange the $\lambda_{i}$ 's in decreasing order and take the first $n$ of them. Then

$$
\left\|\sum_{i=1}^{n} y_{i} \lambda_{i}-y\right\|=\left\|\sum_{i=n+1}^{N} y_{i} \lambda_{i}\right\| \leq \sum_{i=n+1}^{N} \lambda_{i}=S .
$$

We need to prove that $S \leq(N-n) / N$. Assume the opposite. Then

$$
1=\sum_{i=1}^{N} \lambda_{i}>\sum_{i=1}^{n} \lambda_{i}+\frac{N-n}{N}
$$

hence $n / N>\sum_{i=1}^{n} \lambda_{i} \geq n \lambda_{n}$ and $1 / N>\lambda_{n}$. Thus,

$$
S=\sum_{i=n+1}^{N} \lambda_{i} \leq \lambda_{n}(N-n)<\frac{N-n}{N},
$$

which is a contradiction.

So,

$$
S \leq \frac{N-n}{N}=1-\frac{n}{\left[\frac{2}{\varepsilon}\right]+1} \leq 1-\frac{\varepsilon n}{2+\varepsilon}
$$

and the proof of the lemma is finished.

Lemma 6.7. If $X=C(K)$ for a compact Hausdorff space $K$ without isolated points, then for every $\varepsilon$ and $n, \operatorname{Daug}_{n}(X, \varepsilon) \leq 2 / n$. Hence $D_{X}(\varepsilon)$ is of order $\varepsilon^{-1}$.

Proof. Let $x$ and $y \in S(X)$ be arbitrary. Without loss of generality, assume that $x$ attains the value 1 . Take an open neighbourhood $U$ such that $x(u)>$ $1-\varepsilon$ for all $u \in U$. Now pick $n$ disjoint subneighbourhoods $V_{1}, \ldots, V_{n}$ inside $U$. For each of them choose a positive function $\varphi_{i}$ supported on $V_{i}$ such that 
$\left\|\varphi_{i}\right\| \leq 2,\left\|y+\varphi_{i}\right\| \leq 1$ and $y+\varphi_{i}$ attains the value 1 in $V_{i}$. Obviously, $\left\|x+y+\varphi_{i}\right\|>2-\varepsilon$, hence, $y+\varphi_{i} \in l^{+}(x, \varepsilon)$. On the other hand,

$$
\left\|\frac{1}{n} \sum_{i=1}^{n}\left(y+\varphi_{i}\right)-y\right\|=\left\|\frac{1}{n} \sum_{i=1}^{n} \varphi_{i}\right\| \leq \frac{2}{n},
$$

which proves the lemma.

One can show that the same estimates for the $\operatorname{Daug}_{n}$ constants are valid for rich subspaces of $C(K)$-spaces (see [6] for this notion), for vector-valued $C(K)$ - or $L_{1}$-spaces and for spaces of weakly continuous vector-valued functions with the sup-norm.

Remark 6.8. For every Banach space $X$ the constants $D_{X}(\varepsilon)$ can be estimated from below by $(2+2 \varepsilon) /(3 \varepsilon)$, which is bigger than $2 /(3 \varepsilon)$. So the estimates from above which we have for $L_{1}$ and $C$ are of optimal order.

Proof. Suppose $D_{X}(\varepsilon)=n<(2+2 \varepsilon) /(3 \varepsilon)$ for some $\varepsilon>0$. This means in particular that for a fixed element $x \in S(X)$ (taking $y=-x$ ) there are elements $y_{1}, \ldots, y_{n} \in(1+\varepsilon) B(X),\left\|x+y_{n}\right\|>2-\varepsilon$ and $a_{1}, \ldots, a_{n} \geq 0$, $\sum_{k=1}^{n} a_{k}=1$, for which $\sum_{k=1}^{n} a_{k} y_{k}=-x$. Without loss of generality we may assume that $a_{1} \geq 1 / n$ (otherwise just change the enumeration). Plugging in $\left\|x+y_{1}\right\|>2-\varepsilon$ and $x=-\sum_{k=1}^{n} a_{k} y_{k}$ we obtain

$$
\begin{aligned}
2-\varepsilon<\left\|x+y_{1}\right\| & =\left\|y_{1}\left(1-a_{1}\right)-\sum_{k=2}^{n} a_{k} y_{k}\right\| \\
& \leq(1+\varepsilon)\left(1-a_{1}\right)+(1+\varepsilon)\left(1-a_{1}\right) \\
& \leq 2(1+\varepsilon)(1-1 / n) \leq 2-\varepsilon,
\end{aligned}
$$

which is a contradiction.

\section{REFERENCES}

[1] Y. Abramovich. New classes of spaces on which compact operators satisfy the Daugavet equation. J. Operator Theory 25 (1991), 331-345.

[2] B. BeAuzamy. Introduction to Banach Spaces and their Geometry. North-Holland, Amsterdam-New York-Oxford, second edition, 1985.

[3] D. Bilik, V. M. Kadets, R. V. Shvidkoy, G. G. Sirotkin, and D. Werner. Narrow operators on vector-valued sup-normed spaces. Preprint, 2001.

[4] P. Harmand, D. Werner, and W. Werner. M-Ideals in Banach Spaces and Banach Algebras. Lecture Notes in Math. 1547. Springer, Berlin-Heidelberg-New York, 1993.

[5] V. M. Kadets, R. V. Shvidkoy, G. G. Sirotkin, and D. Werner. Banach spaces with the Daugavet property. Trans. Amer. Math. Soc. 352 (2000), 855-873.

[6] V. M. Kadets, R. V. Shvidkoy, And D. Werner. Narrow operators and rich subspaces of Banach spaces with the Daugavet property. Studia Math. (to appear).

[7] T. OikhBerg. The Daugavet property of $C^{*}$-algebras and non-commutative $L_{p}$-spaces. Positivity (to appear).

[8] P. Wojtaszczyk. Some remarks on the Daugavet equation. Proc. Amer. Math. Soc. 115 (1992), 1047-1052. 
Faculty of Mechanics and Mathematics, Kharkov National University, PL. Svobody 4, 61077 Kharkov, Ukraine

Faculty of Mechanics and Mathematics, Kharkov National University, PL. Svobody 4, 61077 Kharkov, Ukraine

E-mail address: vishnyakova@ilt.kharkov.ua

Department of Mathematics, University of Missouri, Columbia MO 65211

E-mail address: shvidkoy_r@yahoo.com

Department of Mathematics, Freie Universität Berlin, Arnimallee 2-6, D-14 195 Berlin, Germany

E-mail address: werner@math.fu-berlin.de 